\title{
Mathematics Education Students' Understanding of Binomial Series Expansion Based on the APOS Theory
}

\author{
Benjamin Tatira ${ }^{1^{*}}$ \\ 1 Walter Sisulu University, SOUTH AFRICA
}

Received 1 July 2021 - Accepted 6 October 2021

\begin{abstract}
Undergraduate students study the topic of binomial series expansion as part of their Calculus course. The purpose of this study was to explore the mental constructions of binomial series expansion of a class of 159 students. Data were collected through a written assessment task by each member of the class. A convenient sample of eleven students from the class was selected to undertake semi-structured interviews. The Action-Process-Object-Schema theory which was used depicts development of understanding of mathematics concepts through the hierarchical growth of mental constructions. Students in the study coped well with questions requiring action-level engagements but grappled with those calling for higher-level mental constructions, namely, processes and objects. Developing an object conception for a mathematical concept is the pinnacle for all teaching but also the most difficult to develop. Thus, this study underlines the significance of helping students construct object conceptions in binomial series expansion.
\end{abstract}

Keywords: APOS theory, binomial series, binomial theorem, calculus, mathematics education, mental structures

\section{INTRODUCTION}

Building upon the quadratic and cubic expansions which students encountered in high school, the binomial series expansion broadens students' understanding binomial expansion to higher powers, and negative and fractional exponents. This series expansion and its generalisations is useful in solving problems in Calculus and Algebra and also serve as an entry point to the calculus of power series. It is important for undergraduate students to use prior of knowledge of binomial expansions of positive whole numbers to develop an understanding of expansions involving fractional and negative exponents. The concept of binomial series provides that link, and serves as an introduction to the calculus of power series. Binomial is also directly connected to geometric series which students have covered in high school through power series. The conditions for convergence is the same for binomial series and infinite geometric series, where the common ratio must lie between -1 and +1 .

Research into the learning of Binomial series has not received enough attention based on available recent literature. There is a dearth of research studies on how undergraduate students conceptualise binomial series expansion. This far, there has not been empirical studies on students' understanding of Binomial series expansion using Action-Process-Object-Schema (APOS) theory or other theories. The main issue in this paper is how undergraduate students conceptualise learning of the Binomial series expansion. Students' mental structures are a key determinant of the extent to which they learn specified topics in mathematics (Ndlovu \& Brijlall, 2019). I assume that a fully developed schema in a given topic has the potential to assist students to answer any given question in that topic and to apply what they would have learnt to unfamiliar contexts. This includes students constructing connections to other mathematics concepts in the same or other topics. I now asked these research questions:

1. Which are the mental constructions that high school students reveal when solving tasks on binomial series?

2. How do students perform on selected assessment items in Binomial series in the context of APOS theory? 


\section{Contribution to the literature}

- To raise lecturers' awareness to the different stages of students' conceptualisation and understanding about binomial series expansion through the APOS framework.

- The study revealed the need to develop process and object mental constructions as students learn a mathematics since the majority of students in this study operated at the action-level of conception.

- This study has the potential to generate more knowledge and literature on students' mental constructions in Binomial series. There are very few empirical studies in this regard.

My interactions with second-year students in an introductory Calculus course inspired this study. Getting to know students' (im)perfections in binomial series during the course of instruction goes a long way to help attain best practices on the part of the instructor. Given the dearth of empirical studies on Binomial series expansion, this study has the potential to generate more knowledge and literature on students' mental constructions in learning Binomial series expansion.

\section{LITERATURE REVIEW}

Literature search for studies on either on Binomial theorem or Binomial series does not show much. Flusser and Francia (2000) wrote a theoretical paper on the history of the Binomial theorem from its inception in the times of Euclid to Newtonian mathematics. Their goal was to derive both the Binomial and multinomial theorems with the aid of visuals, for teaching senior high school and first two years of undergraduate mathematics. Starting with the specific expansion of $(a+b)^{2}$ as expounded in Euclid's elements Book II, they ended with a general formula for the expansion of $(a+b)^{n}$. Finally, the authors gave the origin and derivation of the multinomial theorem for positive integers $m$ and $n$ : $\left(x_{1}+x_{2}+\cdots+x_{m}\right)^{n}$. The study added more literature on the origin and derivation of the binomial theorem; the binomial series expansion was not derived but it was treated as an off-shoot of the binomial theorem. This was done by substituting $n$ with -1 in the formula for the expansion of $(1+x)^{n}$ and no explanation was provided for the change from finite to infinite expansion.

The basis for the binomial series expansion was aptly ascribed to power series, in particular the Taylor series and its variant, the Maclaurin series in a study by TinLam (2003). Having done that, Tim-Lam (2003) then provided an alternative way to teach binomial series to pre-university students in the Singaporean mathematics curriculum. The alternative way was necessary because binomial series is oftentimes taught before advanced calculus in pre-university mathematics. This necessitated the algebraic approach to the binomial series, whose basis is the quadratic expansion and the binomial theorem. He managed to lay bare the underlying meaning of binomial series expansion and the relevance of infinite series whilst skirting the rigour of power series. As a theoretical paper, Tim-Lam (2003) only went as far as expound how to teach binomial series without engaging power series to a class. I did teach using that approach for similar reasons to Tim-Lam (2003); this topic is scheduled to be taught as a sequel to binomial theorem to avoid the power series approach. Inadvertently, Tim-Lam (2003) posited that the typical instructional strategy of binomial series is to present it as a formula to be memorised. This is to be followed by lots of examples and applications of this formula to drill the concept. However, done this way, learning the binomial series becomes a skill of applying rules to achieve the topic learning outcomes. Moreover, the shift of the formula from finite to infinite for non-positive exponents is intuitively committed to memory without meaning. The procedural approach commonly used by instructors at university potentially deprives students the muchneeded deeper understanding of the binomial series concept. In most cases, students may be able to solve problems in binomial series but still lack the total understanding thereof.

In both papers above, the binomial theorem and series were approached from the algebraic standpoint. Tin-Mal (2003) actually acknowledged that Calculus is the rational starting point in deriving the binomial series formula. However, for pre-university students, they would not yet have done the calculus of power series and tests for convergence at the time of introducing binomial series. Consequently, the two authors introduced binomial series algebraically by relating to combinatorics, the Pascal triangle and the binomial theorem. I had to teach binomial series to my class in the same fashion for the same reason. Given such a scenario, it becomes mandatory for me to conduct a study to ascertain the level of students' understanding of the concept of binomial series through the lenses of the APOS theory. Literature search did yield empirical APOS theory studies of students' conceptualisation of binomial series. This represents a gap in literature which this study sought to fill. However, APOS studies were conducted in students' learning other concepts, for example, the Cramer's rule (Ndlovu \& Brijlall, 2019), eigenvectors (Salgado \& Trigueros, 2015), derivatives (Maharaj, 2013; Moru, 2020), trigonometric ratios and their inverses (Martínez-Planell \& Delgado, 2016), the inverse matrix method (Kazunga \& Bansilal, 2020), and others. 


\section{THE APOS THEORY}

\section{Theoretical Framework}

The APOS theory is a constructivist framework with a focus on individual students' mental constructions of mathematical knowledge, which extends Piaget's notion of reflective abstraction to undergraduate mathematics students. Asiala et al. (1996) describe mathematical knowledge in the following way:

An individual's mathematical knowledge is her or his tendency to respond to perceived mathematical problem situations by reflecting on problems and their solutions in a social context and by constructing or reconstructing mathematical actions, processes and objects, by organizing these in schemas to use in dealing with the situations. (p. 32)

I chose the APOS theory on the basis that it focusses on how students construct mathematical knowledge and how to use this information to suggest instructional strategies that can maximise and stimulate learning of a mathematical concept (Salgado \& Trigueros, 2015). The APOS theory enables researchers-cum-instructors to compare differences among students who learn through step-by-step external cues (Action conception); those who work mentally by internalising actions (Process conception); and those who are able to apply the concepts in the solution of new problems and relate current concept to other concepts (Object conception).

According to the APOS theory, when an individual encounters a new mathematical concept, the formation of a concept begins with transformations of existing mental (or physical) objects. These first-formed transformations are called actions. At the action level, students need external cues to carry out a transformation (Dubinsky, 1990). Actions are external as each step in the learning process needs to be articulated step-by-step guided by external instructions. Repeating and reflecting on actions propels students from relying on external cues to having control over them. With this, as students work mentally, they may skip some steps, predict solutions and can work in reverse.

If students become aware of a process in totality, acknowledge that transformations can act on that totality and can construct such transformations physically or mentally, then they have attained cognitive object conception under consideration (Dubinsky et al., 2005). Student can then act on the new constructions of mathematical concepts with higher-level actions and processes (Arnawa et al., 2007). APOS instructional approaches have been successful in helping students to attain an improved understanding of some mathematical concepts (Dubinsky \& McDonald, 2001). Whilst the action, process and object mental structures outline the manner wherein individuals construct a single transformation, a mathematics topic postulates many actions, processes and objects that need to be coherently organised. This postulation is termed schema, which is an individual's collection of actions, processes and object, and other schemas which are connected to the current schema by some general principle. A schema provides a student with a way of handling a mathematical problem situation (Dubinsky et al., 2005). In the case of binomial series, it is the schema that help students to see a binomial series in a given mathematical or real-world situation. A student decides on appropriate mental processes to use in dealing with problem situations and applications concerning binomial series expansion (Borji et al., 2018).

\section{The Genetic Decomposition}

APOS theory uses a hypothetical model to design instructional materials and/or evaluate students' failures or successes in dealing with mathematical problems situations called a genetic decomposition (GD) (Arnon et al., 2014). A GD outlines the mental structures that an arbitrary student needs to construct in order to learn a specific mathematical concept. I have used my experiences on teaching and learning of binomial series, my content knowledge on binomial series and previously published articles on APOS theory to draw the preliminary GD for this study. It is preliminary because it has not been tested and validated. Furthermore, a GD encompasses the description of how the actions, processes and objects are related and organised into a coherent mental structure called schema. The GD is key in APOS studies, which informs instruction of the mathematical concept and the gathering of data for the study. These interrelationships and intricacies are illustrated in Figure 1.

The two schemas indicated at the top are assumed to be present when students commence to study binomial series expansion. These two are coordinated into the binomial series schema. This schema comprises of competencies, concept, mental structures and the indicators for each of the mental structures identified. Students' competencies in the concept of binomial series expansion were categorised as doing the actual expansion, determining the conditions for validity and doing approximations.

\section{METHODOLOGY}

This study was qualitative, which sought to explore second-year undergraduate students' understanding of binomial series expansion in a Calculus course at a South African university. The use of qualitative methodology is "exploring and understanding the meaning individuals or groups ascribe to a social or human problem" (Creswell \& Creswell, 2018, p. 333). The students were registered for a secondary-school fouryear mathematics education degree. The full class of 159 


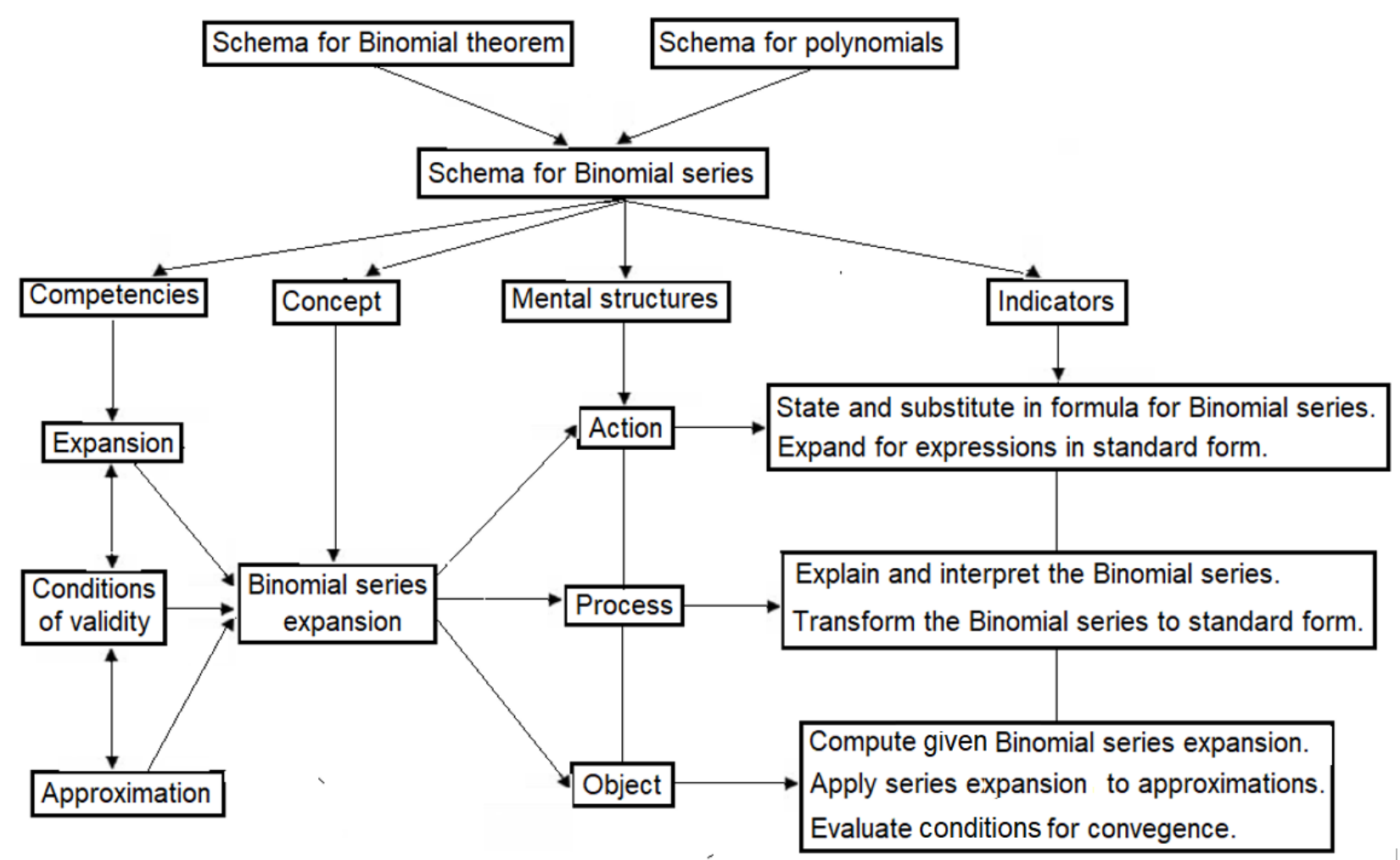

Figure 1. The preliminary GD for binomial series expansion according to the APOS theory

students took part in the study and the researcher was the instructor for the course. The students' age range was 19 to 22. Informed consent was obtained from the participants and ethical clearance protocols were followed. Traditional teaching of binomial series expansion was conducted and guided by the preliminary GD. The students had previously been taught binomial expansion using the Pascal's triangle and the binomial theorem. Each of the 159 students' written responses to an assignment constituted the first set of data. The assignment had two questions on binomial series expansion. Question 1 required students to find the first three terms of the expansion $\sqrt[4]{1+x}$ and then compute the approximate value of $\sqrt[4]{17}$. In question 2 , students were required to find the first three terms of the expansion $(2+x)^{-3}$ and also the values of $x$ for which the expansion is valid. For anonymity, the participants were randomly coded S1, S2 and so on up to S159. Secondly, further nine students were purposively selected for the semi-structured interviews which were audio-taped. The nine consisted of three below average, three average and three above in order to strike a balance of student performance. The interviews delved deeper into students' mental constructions in binomial series expansion, hence only clarification questions were posed. The interview questions depended on the nature of students' responses to the assignment, hence the assignment had to be marked first before the interviews started. Thus, the interviews took place two weeks after the assignment. Data collection took place at the height of the COVID-19 pandemic; the assignment was submitted electronically and scored manually whilst the interviews were conducted telephonically and recorded. The interviews sought to access the full range of students' understanding so that I investigate every written response that appeared from a small percentage of the population (Asiala et al., 1996).

The data from the assignment were coded and this was done according to the coding system by Asiala et al. (1997). This was used to grade the participants' written responses on appropriate scale as follows: categories for no response, category for responses that show some progress towards solution but far from the correct solution, category for almost correct responses with minor flaws in the solution and category for totally correct responses. The content analyses of the same written responses were also done to complement the coded categorical analyses. The interview transcriptions were analysed to identify the degree of their understanding pertaining to the action, object and object mental constructions, informed by the preliminary GD. This way, the students' responses were regarded as a source of the communication intended to convey their understanding of expanding using binomial series (Cohen et al., 2011).

\section{RESULTS}

I counted and tabulated the frequencies of responses for each category. This was followed by an in-depth content analysis of the students' written responses and the transcripts of interviews. This was done to determine evidence of the Action, Process and Object level 
Table 1. Frequencies for question 1 responses

$\begin{aligned} & \text { Categories } \\ & \text { Incorrect responses } \\ & \begin{array}{l}\text { Partially correct responses } \\ \text { Correct binomial series expansion and wrong approximation of } \sqrt[4]{17} \\ \text { Wrong binomial series expansion and correct approximation of } \sqrt[4]{17}\end{array} \\ & \begin{array}{l}\text { Correct response } \\ \text { Total }\end{array} \\ & \qquad(+x)^{\frac{1}{4}}=1+\frac{1}{4}(x)+\frac{1 / 4\left(\frac{1}{4}-1\right)}{2 !} x^{2}+\cdots \\ & \end{aligned}$

Figure 2. A correct method used but with a wrong coefficient of $x^{2}$ by S54

reasoning embedded in the responses (Kazunga \& Bansilal, 2020) in accordance to the preliminary GD.

\section{Question 1 Results}

The frequencies for question 1 responses appear in Table 1.

The attempt rate was relatively high as only three students left the question blank. Students who attempted this question and failed to get correct answers for both the expansion and approximation were nine. In most cases, students who are clueless operate at the preaction conception of binomial series. Of those, four students failed to get the correct coefficient of the term in $x^{2}$ after expanding the expression using the correct method. Their main challenge was in the simplification of the coefficient of the term in $x^{2}$, which was the result of manipulation errors in the process of simplifying the coefficient. The first and second terms were computed with easy and this represented action conception to binomial series expansion. Figure 2 illustrated this situation.

Three students skirted using the binomial series expansion by attempting to apply the law of indices, but in an inappropriate way. For example, S42 expressed

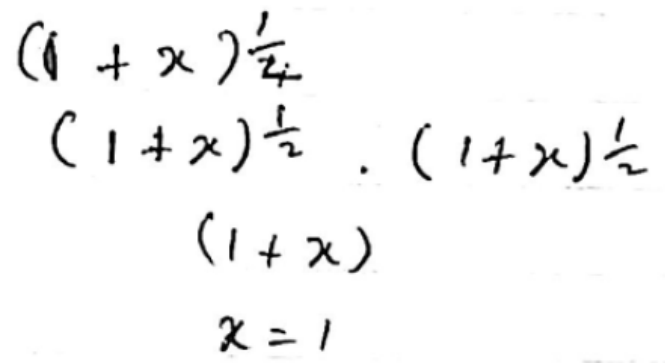

Figure 3. Incorrect application of laws of exponents in expansion by $\mathrm{S} 42$

$(1+x)^{\frac{1}{4}}$ as $(1+x)^{\frac{1}{2}}(1+x)^{\frac{1}{2}}$, which was then simplified to $(1+x)^{1}$. As if it was an equation, they solved and obtained $x=1$ as the final answer (see Figure 3). Again, this is evidence that S42 had not developed action conception of binomial series as he did not even use the formula for binomial series in the solution.

Also, S84 first stated the formula for the binomial series but mixed the terms $n, x$ and $a$ as shown in Figure 4.

In the end, S84 was awfully wrong in the given expansion. The two illustrations are evidence of action conception of binomial series expansion.

$$
\begin{aligned}
(1+a)^{n} & =1+n a+\frac{n(a-1)}{2 !}+n(a-1)(a-2) \\
(1+x)^{\frac{1}{4}} & =1+\frac{1}{4}(x)+\frac{\frac{1}{4}(x-1)}{2 \times 1}+\frac{1}{4}(x-1)(x-2) \\
& =1+\frac{1}{4} x+\frac{x-1}{2}+\frac{3}{3}+\frac{3}{2}\left(x^{2}-3 x^{2}+2\right)
\end{aligned}
$$

Figure 4. A mix-up in the formula of the binomial series by S84 
On approximating the value of $\sqrt[4]{17}$, almost all the participants managed to express $\sqrt[4]{17}$ as $2\left(1+\frac{1}{16}\right)^{\frac{1}{4}}$ but erred afterwards. They indeed realised that the binomial expression must be represented in the standard form of $(1+x)^{n}$ where $|x|<1$. Converting the fourth root of 17 to standard form is an example of object conception of binomial series expansion. After that, students could then apply action and process to the new expression to get the expansion. Substituting in the formula for expansion is action and imagining the infinite terms of the expansion but then taking only the first few terms to approximate is process mental construction. Three students who initially chose to express $17^{\frac{1}{4}}$ as $(1+16)^{\frac{1}{4}}$ later realised their mistakes and subsequently recovered as they now chose the expected expression $(16+1)^{\frac{1}{4}}$. This question was posed to probed whether students could predict the two numbers that would form a binomial expression, without going through the actual steps. This was done to infer whether they had developed Process conceptions of the binomial series. The interview with $\mathrm{S} 11$ alludes to this:

I: Suppose you are asked to find fourth root of 17 . Which two numbers do you choose to make it a binomial expression?

S11: I will choose $18-1$

I: Can you see that you end up finding the fourth root of 18 ! Is that going to be a whole number?

S11: Ooh, I hear. I will choose $16+1$.

Nevertheless, after correctly obtaining $2\left(1+\frac{1}{16}\right)^{\frac{1}{4}}$ these three did not know how to proceed. Their understanding therefore lacked totality as expected at the object conception. Some students in this category made attempts to express the fourth root of 17 as a binomial expression but made some mistakes in the process. In at least two cases, there was no indication of where $\frac{1}{16}$ came from in the expression $1+\frac{1}{16}$. Generally, they jumped to put $x=\frac{1}{16}$ in the expression and the leading 2 was missing. Thereafter they performed a binomial series expansion presumably correct but missing the leading factor 2 . This indicates that the actions of expanding using the binomial series did not interiorise into a process conception.

The partially correct responses comprised of students who could expand the binomial expression but fail to do the approximation, as well as those who failed to expand but were able to do the approximation. From Table 1, more students found it easier to expand a given expression than to find the approximation. Armed with the formula for binomial series, expanding an expression is a step-by-step process; but for approximations,

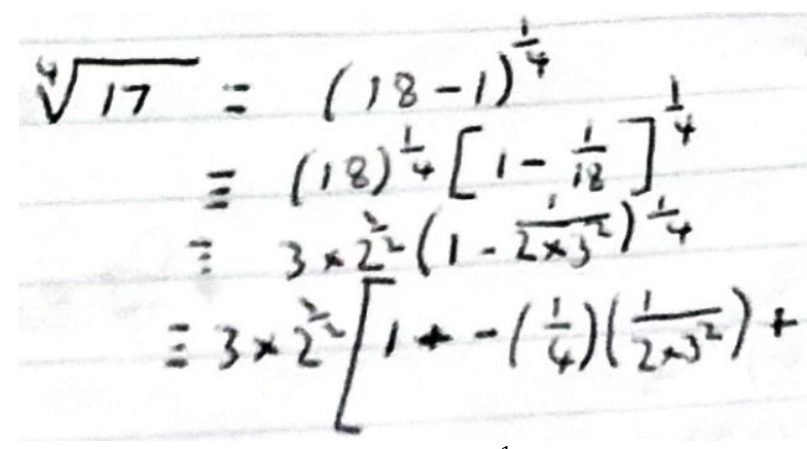

Figure 5. Expanding using $(18-1)^{\frac{1}{4}}$ by S6

students have to think and choose the appropriate value of $x$ that will lead to the required approximation. This is an indication of the process conception, which the students attain. Moreover, the choice of $x$ must comply to $|x|<1$ for the binomial series expansion to be valid. In this study, not many students could do that with ease. In Figure 5, S6 failed to get the correct choice of the two numbers to give 17 .

In this category, eleven students skipped the second part of the question despite having had done the binomial series expansion correctly. This is evidence of failed interiorisation of concept of binomial series. Student S158 gives a reason for skipping the question on expansion of $\sqrt[4]{17}$ :

\section{I: And where is question 1.2?}

S158: [reads the question aloud] It's very challenging also this one.

I: So you decided to skip it.

S158: I didn't want to copy from others. So I thought I should leave it like that.

I: You are right you don't have to copy. But with all the videos that I created and all the lectures I taught, you skip questions and say you don't know.

S158: No, it's not fair for you.

\section{I: What about now? Are you able to do 1.2?}

S158: No sir, I haven't practiced.

Moreover, the difference in performances in the expansion and in approximation was huge. Question 1.1 was step-by-step (action conception) while 1.2 was not (it involved process and object conceptions). Also, 22 students substituted $x=\frac{1}{16}$ in the binomial expansion, without showing clues of where that came from. Two students who attempted to show where $x=\frac{1}{16}$ came from got stuck at the expression $\sqrt[4]{\frac{17}{16}}$. This required object conception to take the split the fourth root and 


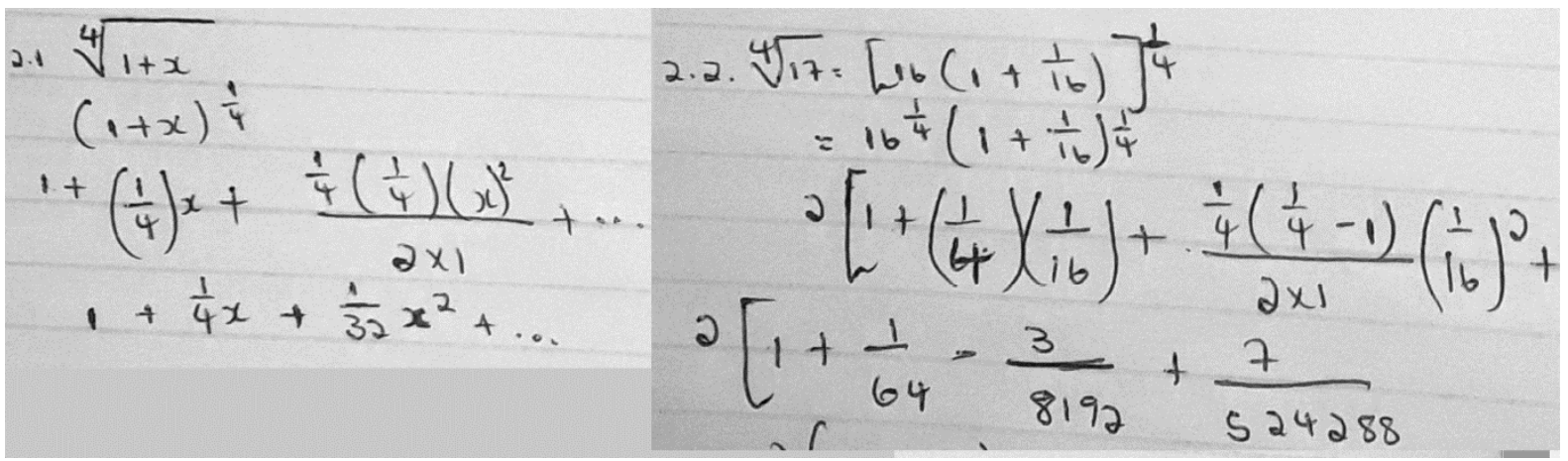

Figure 6. Error in the binomial series expansion which was corrected in the approximation by $\mathrm{S} 45$

then multiply both sides by 4 (in the denominator) to arrive the expected expansion, which the two students could not do. Four students managed to go past this stage but then slipped and got $4\left(1+\frac{1}{16}\right)^{\frac{1}{4}}$.

Concerning the second partial, only seven students amazingly got the approximation correct against the backdrop of incorrect binomial expansion. This scenario depicts importance of understanding prerequisite concepts in constructing the subsequent concepts. Comparatively, it was shown that 55 students successfully conceptualised binomial series expansion but failed to get the approximation correct. All the seven except one had made some attempts to expand but failed. Students like S126 skipped the question on expansion completely. For three students, the only mistake which they made was to mix-up the coefficient of the term in $x^{2}$ in the expansion. S45 fell into this category, whereby she made a mistake in the coefficient of $x^{2}$ but managed to recover and calculated the approximation correctly (see Figure 6). And, S120 and S13 somehow simplified $\sqrt[4]{1+x}$ to $(1+x)^{4}$ and expanded it using the binomial theorem expansion.

Of noteworthy, 85 students (53\%) managed to answer this question correctly, in terms of both the series expansion and the subsequent approximations. The first three terms of the expansion were given based on the correct binomial series formula as expected. On the approximation, the majority of students started with $(16+1)^{\frac{1}{4}}$ which was then simplified to $16^{\frac{1}{4}}\left(1+\frac{1}{16}\right)^{\frac{1}{4}}$ as shown Figure 6. These then simply substituted in the previously expanded expression for $(1+x)^{\frac{1}{4}}$ to get the correct answer. These responses illustrate that the 85 students had the object conception of the binomial series expansion. Nonetheless, three students did not treat the approximation question as a hence, so that they had to start the binomial expansion of $\left(1+\frac{1}{16}\right)^{\frac{1}{4}}$ all over. The dialogue with S124 illustrates this scenario, which led to duplication of work:

I: On question 1.2, you expanded again. Why? Is it not a hence question?
S124: Yes, it is.

I: Then why didn't you just substitute in the expansion from 1.1.

\section{S124: Is that worthy 4 marks?}

I: You are allowed to use the result from a) in other parts of the same question regardless of the mark allocation.

\section{S124: I see.}

I: As a result, you did the expansion two times.

\section{S124: Ok.}

The dialogue with S124 shows that he was more concerned with the mark allocation than using the previously calculated answer. Even though S124 did not see the shorter method, being able to do the expansion again correctly is indicative of object stage reasoning. He understood the concept of binomial series expansion in totality. Two students initially started the approximation with $(1+16)^{\frac{1}{4}}$ but they subsequently recovered from this awkward situation whereby the expansion was not convergent since $16>1$. Re-doing the approximation in the correct way points to action conception.

\section{Question 2 Results}

In this section, I present empirical evidence of students' conceptions of binomial series for the second question.

The frequency of those who skipped this question was 16, actually more than those who presented perfectly correct solutions. This represents students who could not find the required approach to this series expansion and also made no attempts at determining the condition for convergence. These 16 did not display evidence of schema of binomial series expansion. A total of 68 students attempted this question, but varying kinds of errors which rendered the solutions incorrect. The gross error committed by twelve students was failing to transform the binomial expression to the standard form 
Table 2. Frequencies of question 2 responses

\begin{tabular}{lcc}
\hline Categories & Frequency \\
\hline No response & 16 & 68 \\
Incorrect responses & & 34 \\
Partially correct responses & Correct expansion but wrong condition of convergence. & 29 \\
& Wrong expansion but correct conditions of convergence. & 12 \\
Correct response & & 159 \\
Total & & 159 \\
\hline
\end{tabular}

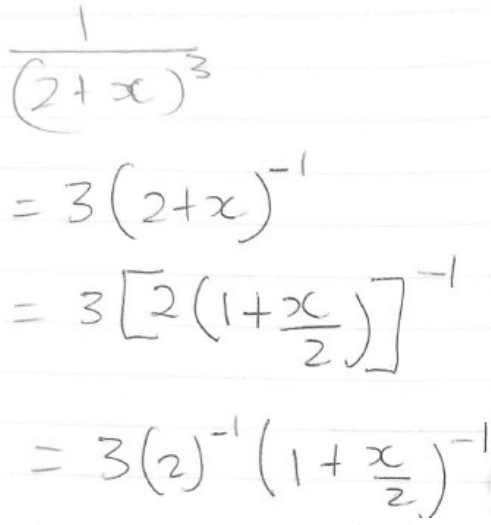

Figure 7. S15's attempts to standardise the binomial expression before expanding

$2^{-3}\left(1+\frac{x}{2}\right)^{-3}$. This represents an encapsulation of binomial series expansion as further actions and processes are required to obtain the final expansion. Figure 7 depicts one such case.
Two students used the Pascal's triangle to expand the expression as shown in Figure 8. This represents preAction conception to binomial series because students ended up using the binomial theorem formula but with negative exponents.

Five students transformed the expression to $\frac{1}{(2+x)^{3}}$ and then attempted to expand the denominator by removing the brackets. And five students somehow replaced the two with a one and expanded, as shown in Figure 9. Both S108 and S1 reflected lack of action conception of binomial series expansion.

Having performed the required standardisation and expanding, 38 students obtained an incorrect coefficient of the term in $x^{2}$. They got 3 instead of the expected $\frac{3}{16^{\prime}}$ which was a result of mistaken simplification. This response illustrates that the 38 students had an action conception of binomial series expansion although they were not able to calculate the coefficient of the term in $x^{2}$. Moreover, eight students expanded using $x$ instead $\frac{x}{2}$

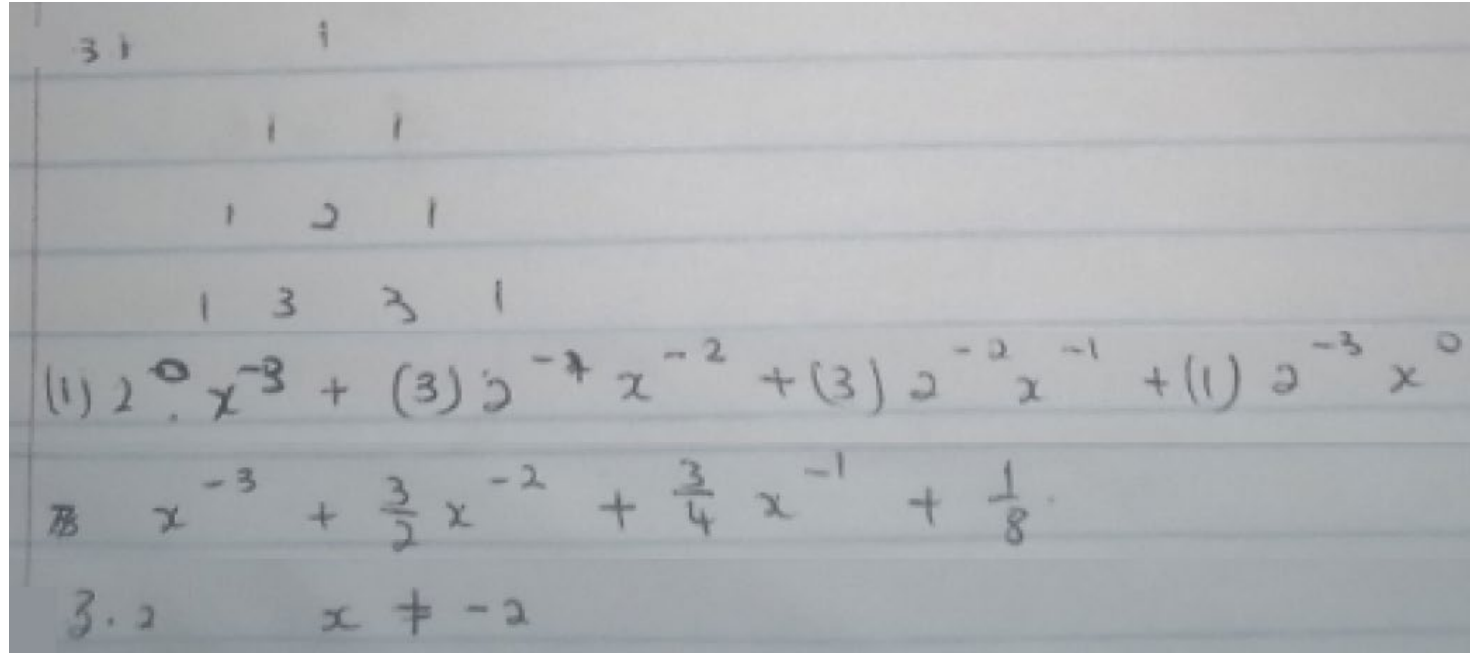

Figure 8. Binomial series expansion done using the Pascal's triangle by S108

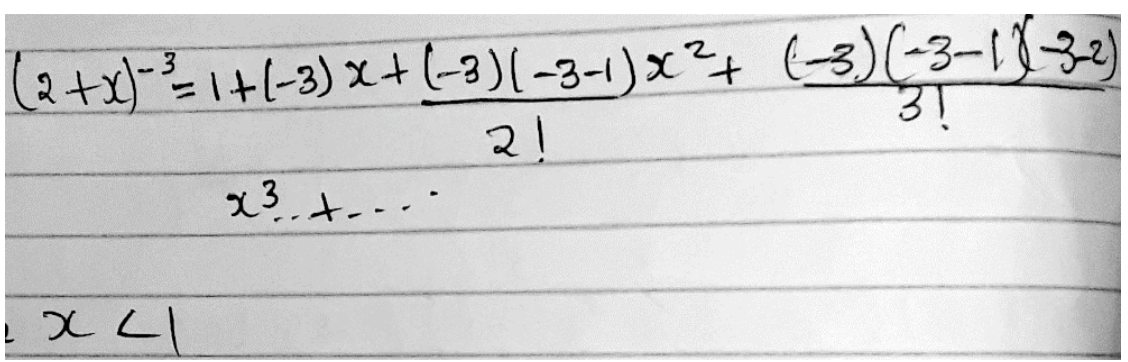

Figure 9. Replacing a two with a one in S1's expansion 


$$
\begin{aligned}
& (2+\mathrm{x})^{-3}=\sum_{k=0}^{-3}\left(\begin{array}{c}
-3 \\
k
\end{array}\right) \times 2^{-3-k}\left(x^{k}\right) \\
& =\left(\begin{array}{c}
-3 \\
0
\end{array}\right)\left(2^{-3}\right)+\left(\begin{array}{c}
-3 \\
1
\end{array}\right) \times 2^{-4} x+\left(\begin{array}{c}
-3 \\
2
\end{array}\right)\left(2^{-5}\right)\left(x^{2}\right) \ldots \\
& =2^{-3}+(-3)\left(2^{-4} x\right)+\left(\frac{-3 \times-4}{4}\right)\left(2^{-5}\right)\left(x^{2}\right)+\cdots \\
& =2^{-3}+\left(\frac{-3}{16}\right) x+\frac{3}{8} x^{2}+\cdots
\end{aligned}
$$

Figure 10. Use of the binomial theorem expansion in an expression with negative exponent by S130

which is a likely case of misunderstood standardisation of the binomial expression. Two students resorted to the binomial theorem to expand the expression and had a strange representation of combination as illustrated in Figure 10.

The expansion by S130 represents lack of action stage reasoning, the first stage in students' construction of the binomial series schema. Students lack necessary process skills to explain a concept without referring to a particular example or solving a problem. S117 could not give a succinct definition of binomial series but could apply it to a given problem. In the written responses, he managed to get question 1 correct but skipped question 2. This is evidence of action skills dominance over process skills.

\section{I: And my first question to you is; what do you understand by the term binomial series?}

S117: I think it refers to the expression or equation whereby I have to solve. I don't know how to explain it but I have a clue.

I: So can you tell me; is it different from the binomial theorem?

S117: Yes. Binomial theorem is used to solve problems where we can't use Pascal's triangle.

\section{I: Again, are the two different?}

S117: They are the same.

The dialogue above with S117 also confirmed that some students treated the binomial theorem and the binomial series as one entity, which is evidence that S117's action did not interiorise to processes. Students had challenges in determining the values for which the series is valid: nine did not answer the question; and a further nine responded with the solution: $-1<x<1$. But $-1<x<1$ is the validity for the basic expansion $(1+x)^{n}$. Eleven students also wrote that it is valid for $0<x<1$. Four students realised that $(2+x)^{-3}$ is equivalent to $\frac{1}{(2+x)^{3}}$, hence concluded that the expression is defined for $2+x \neq 0 \Rightarrow x \neq-2$. Such gross misstatement depicts lack of action conception of binomial series. While this may sound true for the expression to be defined indeed, the condition for validity of binomial series expansion is $\left|\frac{x}{2}\right|<1$. Validity for the convergence of the expansion in this sense is entirely different from the general state of undefined which guards against division by zero.

Similar errors were observed for students who got the expansion right but failed to give the correct region the expansion is valid. The frequency for the first partially correct responses was 34 . However, the number of students who did not respond to the question of defining the valid region was seven. Twelve students also got the solution $-1<x<1$ and eight got $2+x \neq 0$, as was shown above. The category of second partial responses indicated that 29 students were able to determine the correct values where this expansion is defined, despite failing to expand the expression. This attempt suggests students were moving towards the construction of the process conception of the binomial series. Interestingly, some students made mistakes in the written responses but recovered in the verbal responses as shown in the dialogue with S68.

I: The question is asking for the values of $x$ for which the expansion above defined. And you are saying $x<1$ in your written responses. Is that true?

S68: No. I am saying $x$ lies between -1 and 1 . But since our value is $x$ over 2 , then our value of $x$ is $\frac{x}{2}$ and is supposed to between 1 and -1 . Then multiply by 2 and then we get range -2 and 2 .

S68's response show that he was able to reflect in totality the validity of binomial expressions, which is an indication of an object conception. All the 29 students except one made some meaningful attempts at using the binomial series formula to expand. One student skipped the question. Of these, eleven students only made a computational error of the coefficient of the term in $x^{2}$; they got $3 x^{2}$ instead of the expected $\frac{3}{16} x^{2}$. This was 


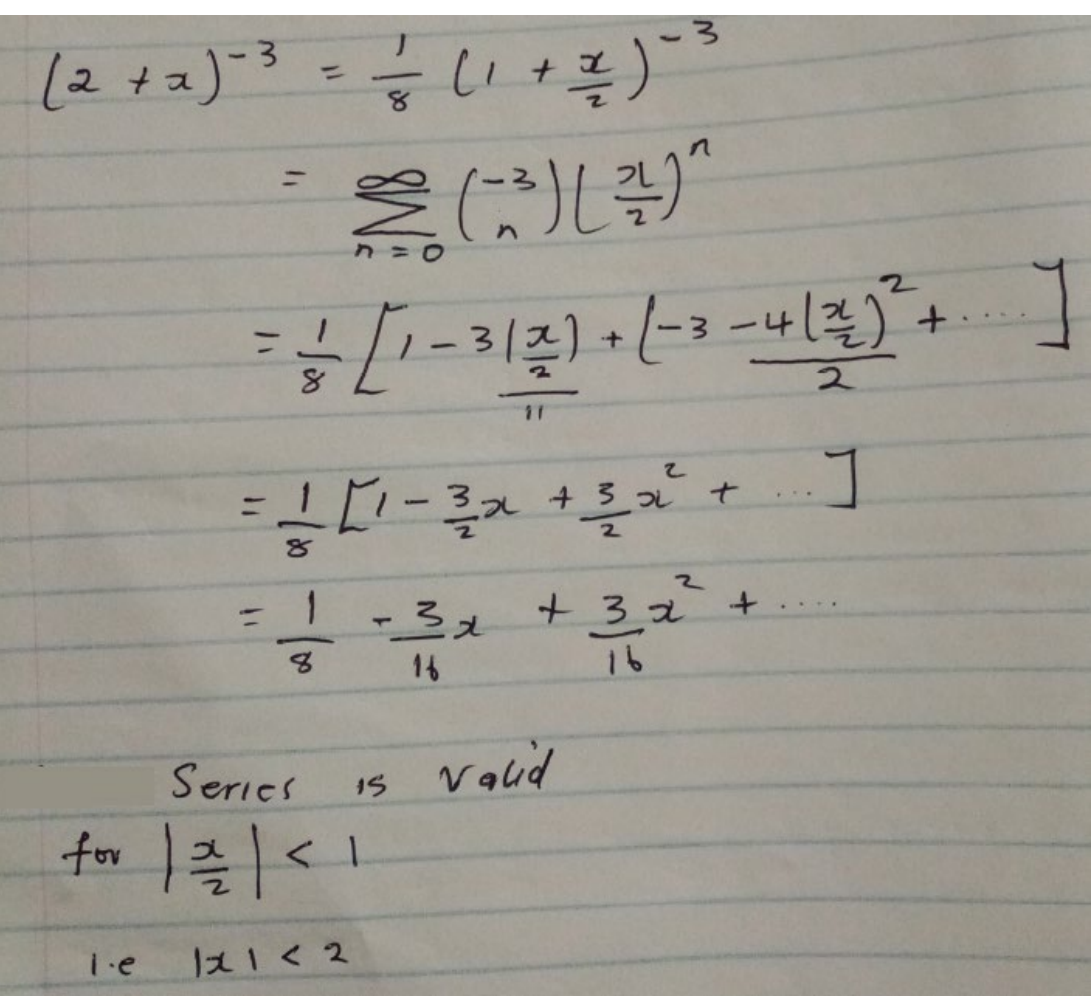

Figure 11. A correct response to question 2 by S73

followed by eight students whose expansion lacked the leading $\frac{1}{8}$. Four more students slipped when they expressed $2^{-3}$ as $\frac{1}{18}$. In essence, most students' main weakness was obtaining the correct coefficient for the term in $x^{2}$; the first two terms were generally straightforward. To complete the analyses, only $13(8 \%)$ students were able to compute both the expansion and the values where the expansion was valid, which suggests these students were able to draw upon object reasoning about binomial series. Most students performed the mental transformation of $(2+x)^{-3}$ to the standardised form $\frac{1}{8}\left(1+\frac{x}{2}\right)^{-3}$, without necessarily writing down. This is evidence of process conception of binomial series. Furthermore, imagining the infinite number of terms can only be understood at the process conception (Areaya \& Sidelil, 2012). After getting the standardised form of the binomial, students follow external prompts in the stepby-step procedure to get the final expansion: $\frac{1}{8}-\frac{3}{16} x+$ $\frac{3}{16} x^{2}+\cdots$. Students managed draw upon object conception of binomial series in order to perform the entire procedure of standardising and applying the formula of binomial series to expand until the final expansion.

To expand, they started with the formula for binomial series expansion and they applied it according to the demands of the question. Most of them started with $-1<x<1$ and contextualised the answer, as shown in the dialogue with S109.

\section{I: Can you show me now the correct values of $x$ ?}

S109: So I think to get the answer, we will use that method of convergence. It says $-1<$ common ratio $r<1$. In the place of $r$ we substitute by $x$ and its coefficient.

I: Why are you saying $x$ ?

S109: So my common ratio; it would be $\frac{x}{2}$.

\section{I: Proceed.}

S109: In place of $r$ we put $\frac{x}{2}$.

I: Yes.

S109: We are looking for values of $x$ so we multiply both sides by 2 and we will get $-2<x<$ 2.

To get the values for which the expansion is valid mainly required students' process skills. Mentally, students can deduce the equivalence of the region of validity to $|x|<1$ in the expression $(2+x)^{-3}$. This is found to be $\left|\frac{x}{2}\right|<1$, which simplifies to $|x|<2$. The correct response for both parts of question 2 is illustrated in Figure 11. Only 12 students managed to obtain this, which is an indication that the majority of the students had not developed a full understanding of binomial series expansion of problems of this nature and determining the region to which it is valid.

Students like S73 relied on their process conception to transform the given expression to standard form, 
necessary for binomial series expansion. This was done mentally as expected at process stage. After this transformation was done, S73 went on to compute the first three terms of expression inside the brackets stepby-step, which represent action conception. But this action stage after the initial transformation depicts encapsulation of binomial series expansion. This student's understanding was entire as he realised that further actions and processes can be applied to the expansion. Furthermore, imagining infinite terms to the expansion (indicated by the three dots) even though he only wrote the first three is a further process conception to the totality of his understanding of binomial series expansion. Lastly, S73 mentally computed the region for validity of the expression, which was a further process conception. By drawing a comparison with $|x|<1$, S73 was able to figure out the correct region $\left|\frac{x}{2}\right|<1$. The entirety of actions and processes represent an encapsulation of the concept of expansion of problems of this sort.

\section{DISCUSSION}

This study explored the undergraduate students' understanding of binomial series expansion in an APOS framework. I attempted to describe the level of students' APOS mental constructions after they learnt the topic of binomial series expansion by traditional instruction. The pre-schemas of binomial theorem and the polynomial were well coordinated to support the binomial series schema development in most cases. This worked as expected for the majority as it led to expected competencies, mental constructions and the indications thereof (according to the preliminary GD). However, for a few students, these old experiences caused some conflicts in learning the concept of binomial series. Results revealed that some students resorted to the Pascal's triangle and the binomial theorem to expand binomial expressions with negative and fractional exponents. Students stuck to the binomial theorem procedural fashion, to the extent of manipulating the combinations for negative $n$, which suggests lack of action conception of binomial series. These conflicts are what Tall (2004) called met-befores, which possibly interfere with later concept development.

Most students in the study were more comfortable with construction requiring straight action-level conceptions. Question 1 was in the expected form to apply binomial series expansion, hence the relative frequency for those who solved this correctly was high. Normally, action conception is application of memorised facts (Martínez-Planell \& Delgado, 2016). Contrariwise, question 2 required to be simplified to standard form first before substitution in the formula became possible. Consequently, the relative frequency for those who got this question correct was low, with many students even skipped this question. Actions as entry-level constructions to any topic are indisputable, but definitely are not sufficient to fully lead to an understanding of a mathematics concept. In this study, I noted students had numerical manipulation problems in computing the coefficient of $x^{2}$ and other higher of powers of $x$, which is sign of lack of interiorisation of the binomial series concept. However, it was evident that the frequency of students honed as the knowledge demands require process and object construction of knowledge. For many students, procedural-learning (action conception) can become a way of life once they fail to compress mathematics procedures into thinkable concepts (Tall, 2004).

Actions were successfully interiorised into processes by some students. The interview revealed that students encountered difficulties in defining the term binomial series without explicitly performing it. Indeed, most students demonstrated their understanding by applying binomial series expansion to a specific problem. Neither could they distinguish binomial theorem from binomial series. Process skills are made manifest when students can imagine that the expansion of binomial series continues indefinitely in their minds (Kolar \& Čadež, 2012). However, a few students could envision this, consequently, they used finite expansion to binomial series expansions.

Approximations and intervals of convergence for binomial series expansions require some thinking before applying actions or processes to them. Thus, they are not procedural as the students have to figure out the appropriate expressions to determine the validity. However, many students attempted the procedural way by arbitrarily choosing to substitute $x$ for $\frac{1}{16}$ into the expression of $(1+x)^{\frac{1}{4}}$ without deriving its origins. By so doing, they missed the leading 2 and marred their subsequent expansion. Good object skills means being able to recognise the pertinent idea at a glance (Moru, 2020) and the applicability of an object in a given problem situation.

The precepts envisaged in the GD were seen in practice as students learnt and were assessed in the concept of binomial series expansion. Armed with the formula for binomial series, students had less difficulties to expand binomial expressions, which is at action-level of conceptualisation. To determine the set where the series is valid, students had to go the beyond step-bystep manipulation of the problem into process skills. They were supposed to interpret the binomial expression and sometimes change it to standard form before they established the set. And this was not an easy feat for many students. Thus, the GD was useful in giving frame to the analyses of data and the discussion of findings (Martínez-Planell \& Delgado, 2016). Students are not always static at one APOS level as they learn a concept. After a short time lapse between the written assessment and the interviews, students had grown in 
knowledge within the same APOS level and across levels. Continual engagement with content as individuals and/or as class with the instructor potentially made that possible.

\section{CONCLUSION AND IMPLICATIONS}

Students in this study coped well with questions requiring action-level engagements but grappled with those calling for higher-level mental constructions, namely, processes and objects. For instance, students could not distinguish the binomial theorem from the binomial series; they used finite expansion to a binomial series expansion. The moment a computation involves an infinite number of steps, it can only be understood through a process mental construction (Areaya \& Sidelil, 2012). In cases where they erred at the action-level, manipulation errors were the major cause. The step-bystep conception of binomial series was certainly present in the students' work, but some simplification and calculation errors marred the overall response. This may suggest many students learn expansion by the binomial series without understanding binomial series, the notion that supports such computations. To help overcome this, students need more opportunities to engage with content at the action level. The students' inability to explain the concept of binomial series mentally and differentiate it from binomial theorem denote weak interiorisation mental mechanism. The approximations of radicals using binomial series is an encapsulation of students' efforts in learning binomial series expansion. However, few students managed to apply actions and processes to the approximations using binomial series, which signify object conception of binomial series expansion. Developing an object conception for a mathematical concept is the pinnacle for teaching but it is also difficult and timeous (Arnon et al., 2014), which was confirmed by findings of this study. Thus, this study underlines the significance of helping students construct object conception of binomial series expansion. Use of APOS theory enable mathematics education researchers to explain divergences of conceptualisation amongst students who operate at the level of facts/formulae and those who can apply concepts in solving new problems (Salgado \& Trigueros, 2015).

Future studies may engage students in nontraditional instruction whereby they may work on plenty of activities in a computer-based learning environment as propounded the Dubinsky (1990). However, there remains logistical challenges for a class of 159 students or more, a common feature in education faculties. Future studies can also focus on a mix of binomial theorem and binomial series expansions so students can have a broader conception and be able to distinguish the two.

This study, being a self- and classroom-based, has been of great help to me in terms of improving my instructional practices in an institution with scant resources. It can also help instructors in similar institutions. In essence, this study highlighted the need to strengthen process and object skills, based on evidence in binomial series expansion. It is my hope that results of this study will contribute to the knowledge of how undergraduate students understand binomial series expansion at all levels of APOS theory.

Funding: No funding source is reported for this study.

Declaration of interest: No conflict of interest is declared by author.

\section{REFERENCES}

Aljohani, S. (2016). History of binomial theory. International Journal of Scientific \& Engineering Research, 7(4).

Areaya, S., \& Sidelil, A. (2012). Students' difficulties and misconceptions in learning concepts of limit, continuity and derivative. The Ethiopian Journal of Education, 33(2), 1-37.

Arnawa, I. M., Sumarno, U., Kartasasmita, B., \& Baskoro, E. T. (2007). Applying the APOS theory to improve students' ability to prove in elementary abstract algebra. Journal of Indonesia Mathematical Society (MIHMI), 13(1), 133-148. https:// doi.org/10.22342 /jims.13.1.80.133-148

Arnon, I., Cottrill, J., Dubinsky, E., Oktac, A., Fuentes, S. R., Trigueros, M., \& Weller, K. (2014). APOS theory: A framework for research and curriculum development in mathematics education. Springer Science \& Business Media. https://doi.org/10.1007/978-14614-7966-6

Asiala, M., Brown, A., DeVries, D., Dubinsky, E., Mathews, D., \& Thomas, K. (1996). A framework for research and curriculum development in undergraduate mathematics education. In J. Kaput, A. H. Schoenfeld, \& E. Dubinsky (Eds.), Research in collegiate mathematics education II (pp. 1-32). American Mathematical Society. https:// doi.org/10.1090/cbmath/006/01

Asiala, M., Cottrill, J., Dubinsky, E., \& Schwingerdorf, K. E. (1997). The development of students graphical understanding of the derivative. Journal of Mathematical Behaviour, 16(4), 399-431. https:/ / doi.org/10.1016/S0732-3123(97)90015-8

Borji, V., Alamolhodaei, H., \& Radmehr, F. (2018). Application of the APOS-ACE theory to improve students' graphical understanding of derivative. Eurasia Journal of Mathematics, Science and Technology Education, 14(7), 2947-2967. https://doi.org/10.29333/ejmste/91451

Cohen, L., Manion, L., \& Morrison, K. (2011). Research methods in education (7th ed.). Routledge. 
Creswell, J. W., \& Creswell, J. D. (2018). Research design qualitative, quantitative, and mixed methods approaches (5th ed.). Sage.

Dubinsky, E. (1990). Teaching mathematical induction II. Journal of Mathematical Behavior, 8, 285-304.

Dubinsky, E., \& McDonald, M. A. (2001). APOS: A constructivist theory of learning in undergraduate mathematics education research. In The teaching and learning of mathematics at university level (pp. 275282). Springer. https://doi.org/10.1007/0-30647231-7_25

Dubinsky, E., Weller, K., Mcdonald, M. A., \& Brown, A. (2005). some historical issues and paradoxes regarding the concept of infinity: An apos-based analysis: Part 1. Educational Studies in Mathematics, 58, 335-359. https://doi.org/10.1007/s10649-005$2531-z$

Flusser, P., \& Francia, G.A. (2000). Derivation and visualization of the binomial theorem. International Journal of Computers for Mathematical Learning, 5, 324. https:/ / doi.org/10.1023/A:1009873212702

Kazunga, C., \& Bansilal, S. (2020). An APOS analysis of solving systems of equations using the inverse matrix method. Educational Studies in Mathematics, 103, 339-358. https:/ / doi.org/10.1007/s10649-02009935-6

Kolar, V. M., \& Čadež, T. H. (2012). Analysis of factors influencing the understanding of the concept of infinity. Educational Studies in Mathematics, 80(3), 389-412. https://doi.org/10.1007/s10649-0119357-7

Maharaj, A. (2013). An APOS analysis of natural science students' understanding of derivatives. South
African Journal of Education, 33(1), 458. https:/ / doi.org/10.15700/saje.v33n1a458

Martínez-Planell, R., \& Delgado, A.C. (2016). The unit circle approach to the construction of the sine and cosine functions and their inverses: An application of APOS theory. Journal of Mathematical Behavior, 43, 111-133.

https:/ / doi.org/10.1016/j.jmathb.2016.06.002

Moru, E. K. (2020). An APOS analysis of university students' understanding of derivatives: A lesotho case study. African Journal of Research in Mathematics, Science and Technology Education, 24(2), 279-292. https://doi.org/10.1080/18117295.2020.1821500

Ndlovu, Z., \& Brijlall, D. (2019). Pre-service mathematics teachers' mental constructions when using Cramer's rule. South African Journal of Education, 39(1), 1-13. https://doi.org/10.15700/saje.v39n1 a1550

Salgado, H., \& Trigueros, M. (2015). Teaching eigenvalues and eigenvectors using models and APOS Theory. Journal of Mathematical Behavior 39, $100-120$

https:// doi.org/10.1016/j.jmathb.2015.06.005

Stewart, J. (2008). Calculus: Early transcendentals (6 $6^{\text {th }}$ ed.). Thomson Brooks/Cole.

Tall, D. (2004). Thinking through three worlds of mathematics. Proceedings of the 28th conference of the International Group for the Psychology of mathematics education (pp. 281-288).

Tin-Lam, T. (2003). On teaching binomial series: more meaning and less drill. International Journal of Mathematical Education in Science and Technology, 34(1), 115-121. https:/ / doi.org/10.1080/002073903 1000158182

\section{http://www.ejmste.com}

